\title{
Calibration of ac and dc magnetometers with a $\mathrm{Dy}_{2} \mathrm{O}_{3}$ standard
}

\author{
D.-X. Chen, ${ }^{1}$ V. Skumryev, ${ }^{1}$ and B. Bozzo ${ }^{2}$ \\ ${ }^{1}$ ICREA and Departament de Física, Universitat Autònoma de Barcelona, 08193 Bellaterra, Barcelona, Spain \\ ${ }^{2}$ Institut de Ciència de Materials de Barcelona, CSIC, 08193 Bellaterra, Barcelona, Spain
}

(Received 29 November 2010; accepted 27 March 2011; published online 27 April 2011)

\begin{abstract}
The ac susceptibility and magnetization curves of a glued $\mathrm{Dy}_{2} \mathrm{O}_{3}$ powder sample are measured by an ac susceptometer and a dc superconducting quantum interference device magnetometer, both of which have been calibrated previously. It is shown that the magnetic moment of the paramagnetic sample as a function of field and temperature may be accurately expressed by a combination of the Curie-Weiss law and the Langevin function at $T>45 \mathrm{~K}$ with three adjusting parameters, so that the dc magnetization curves and the magnitude and phase of ac susceptibility at different values of dc bias field measured by any magnetometer can be calibrated by using $\mathrm{Dy}_{2} \mathrm{O}_{3}$ as a standard. The expressions are empirical and cannot be justified in the entire field and temperature range by existing theories of paramagnetism. Below $10 \mathrm{~K}$, indication of approaching a possible phase transition is found. It is shown that pure $\mathrm{Dy}_{2} \mathrm{O}_{3}$ powder may be used as a primary standard, with susceptibility $[13.28(T+17)]^{-1} \mathrm{emu} / \mathrm{Oe} / \mathrm{g}$ at $T>50 \mathrm{~K}$ and $H<10 \mathrm{kOe}$, in consistency with the Curie-Weiss law and the quantum mechanical theory of paramagnetism. () 2011 American Institute of Physics. [doi:10.1063/1.3581224]
\end{abstract}

\section{INTRODUCTION}

Instruments for magnetic measurements may be named as magnetometers if the magnetic moment of the sample is directly measured by them. The calibration of two Quantum Design magnetometers will be the topic of the present work, which are an ac susceptometer of PPMS (Physical Property Measurement System) and a dc superconducting quantum interference device (SQUID) magnetometer of MPMS (Magnetic Property Measurement System). ${ }^{1-3}$ According to the Quantum Design manuals, a paramagnetic Pd cylinder of mass about $0.27 \mathrm{~g}$ is provided by the factory to calibrate the SQUID dc magnetometer and the extraction dc magnetometer in PPMS at a high field, whose mass susceptibility is $5.25 \times 10^{-6} \mathrm{emu} / \mathrm{Oe} / \mathrm{g}$ at $T=298 \mathrm{~K}$ with uncertainty less than $0.5 \%,{ }^{4}$ and a glued $\mathrm{Dy}_{2} \mathrm{O}_{3}$ powder (i.e., $\mathrm{Dy}_{2} \mathrm{O}_{3}$ powder embedded in epoxy) cylinder is provided by the factory with 1 order of magnitude higher moment susceptibility (magnetic moment per unit field) to calibrate the ac susceptometer. The susceptometer is calibrated using the $\mathrm{Dy}_{2} \mathrm{O}_{3}$ standard sample at $T=298 \mathrm{~K}$, field amplitude $H_{m}=800 \mathrm{~A} / \mathrm{m}$, and frequency $f=1000 \mathrm{~Hz}$, whereas the moment susceptibility of the $\mathrm{Dy}_{2} \mathrm{O}_{3}$ standard sample itself is obtained by measurement at $T=298 \mathrm{~K}$ and $H=800 \mathrm{kA} / \mathrm{m}$ in the extraction dc magnetometer, calibrated by using the Pd standard sample at $T=298 \mathrm{~K}$ and $H=1600 \mathrm{kA} / \mathrm{m}$.

All such recommended calibrations are carried out by measuring the magnetic moment of standard samples at fixed temperature and field, assuming the error of magnetic field to be negligible. This assumption is basically valid in instruments commercially available, whose magnetizing field coils are made accurately with a stable ratio of field to current, so that the field is obtained by measuring the current according to the Ampère law. However, if the coil is a superconducting magnet, the hysteretic magnetization of superconductor itself will give a complicated demagnetizing field in the bore of the magnet, so that the field cannot be measured by the current accurately. This may be a reason why dc magnetometers with superconducting magnet are calibrated at a very high field, at which the demagnetizing effects are negligible. In all such calibrations, temperature and frequency are also assumed to be accurate.

In order to know the performance of the studied ac susceptometer in the entire range of $T, H_{m}$, and $f$, we have calibrated it with a copper cylinder standard of $5 \mathrm{~mm}$ in diameter and length. ${ }^{5}$ Different from conventional calibration techniques mentioned above, the electromagnetic properties of the copper cylinder are unknown and are determined during the calibration of the ac susceptometer. In other words, the calibration is directly based on the Maxwell equations and the Ohm law but is not transmitted from another primary standard sample. Thus, it can be widely adapted by users of any kind of commercial or home-made ac susceptometers. By comparing the magnitude of ac susceptibility measured at $T=10 \mathrm{~K}$ and a number of values of $f$ with the calculated eddy-current susceptibility, we have obtained an averaged magnitude correction factor $k=1.081$ for that particular ac susceptometer. In the present paper, we will use the same ac susceptometer and a dc SQUID magnetometer to measure a glued $\mathrm{Dy}_{2} \mathrm{O}_{3}$ powder cylinder of diameter $5 \mathrm{~mm}$ and length $6 \mathrm{~mm}$ provided by the factory (with unknown $\mathrm{Dy}_{2} \mathrm{O}_{3}$ mass) to show how both magnetometers may be further calibrated by this standard sample. We will also show that a pure $\mathrm{Dy}_{2} \mathrm{O}_{3}$ powder sample with known mass can serve as a primary standard.

Such a calibration will also be made in a large range of $T, H_{m}$, and $f$, for which the actual functional properties of the $\mathrm{Dy}_{2} \mathrm{O}_{3}$ sample have to be expressed accurately. In Sec. II, the Curie-Weiss law is used for the low-field moment susceptibility, with the Curie and the Weiss constants 
determined accurately by ac susceptibility measurements at $T>45 \mathrm{~K}$. The resulting expression is used for the magnitude and phase calibration of the ac susceptometer at different $T$ and $f$ and the low-field susceptibility calibration of the dc magnetometer at different $T$. In Sec. III, the Curie-Weiss law is combined with the Langevin function to describe the $T$ and $H$ dependence of magnetic moment with an additional adjusting parameter, the elementary moment, determined by dc magnetic measurements. The resulting expression for dc field-dependent differential susceptibility is used for calibrating the magnitude and phase of the incremental ac susceptibility at different dc field.

It is found that complex behaviors occur at low temperatures and that the resulting elementary moment is illogically $T$ dependent and larger than that predicted by the quantum mechanical theory, as explained in Sec. IV. However, as described in Sec. $\mathrm{V}$, the measurements of a pure $\mathrm{Dy}_{2} \mathrm{O}_{3}$ powder sample of known mass validate the quantum mechanical theory at low fields so that pure $\mathrm{Dy}_{2} \mathrm{O}_{3}$ may serve as a primary standard, whose low-field susceptibility per unit mass is a function of $T$ only. In Sec. VI, the influence of finite sample dimensions on the sensitivity of moment measurements is calculated to make additional error analysis. Further discussion and the final conclusion are presented in Secs. VII and VIII.

We will use electromagnetic units for the data, since they are widely used in the literature and the data files of both instruments. However, as generally recommended, SI units will be used in basic physical equations. Which units system to be used will be noted in the text. In electromagnetic system, the units of magnetic moment and field are erg/G and Oe, respectively, but the former is written emu customarily in the present paper. In SI, the units of moment and field are $\mathrm{Am}^{2}$ and $\mathrm{A} / \mathrm{m}$, respectively. The conversion between both systems may be made by $1 \mathrm{Am}^{2}=10^{3}$ emu and $1 \mathrm{~A} / \mathrm{m}=4 \pi \times 10^{-3}$ Oe.

\section{CALIBRATION USING CURIE-WEISS LAW}

\section{A. Curie-Weiss moment susceptibility}

The amplitude of complex ac moment $m_{m}=m_{m}^{\prime}-j m_{m}^{\prime \prime}$ of the glued $\mathrm{Dy}_{2} \mathrm{O}_{3}$ sample was, using the incorporated PPMS software, measured at zero dc field, ac field amplitude $H_{m}$ $=10 \mathrm{Oe}, f$ between 11 and $3333 \mathrm{~Hz}$, and $T$ between 2 and $300 \mathrm{~K}$, following a standard procedure after the sample was automatically centered at a certain temperature. ${ }^{1}$ The results of complex ac moment susceptibility $\chi=m_{m} / H_{m}=\chi^{\prime}$ $-j \chi^{\prime \prime}$, after a magnitude correction with $k=1.081$ was applied to $m_{m}$, are shown in Fig. 1 for $f$ $=11,33,111,333,1111$, and $3333 \mathrm{~Hz}$ and $T \leq 40 \mathrm{~K}$. We observe that positive $\chi^{\prime}$ is weakly $f$ dependent and it decreases with increasing $T$, whereas $\chi^{\prime \prime}$ is 2 orders of magnitude smaller than $\chi^{\prime}$ and may be positive or negative with a remarkable $f$ and $T$ dependence.

As a paramagnetic material, the low-field susceptibility of $\mathrm{Dy}_{2} \mathrm{O}_{3}$ sample should follow the Curie-Weiss law, ${ }^{6}$ expressed in SI units by

$$
\left.\frac{d M}{d H}\right|_{H \rightarrow 0}=\frac{n \mu_{0} m_{0}^{2}}{3 k_{B}\left(T-T_{0}\right)},
$$
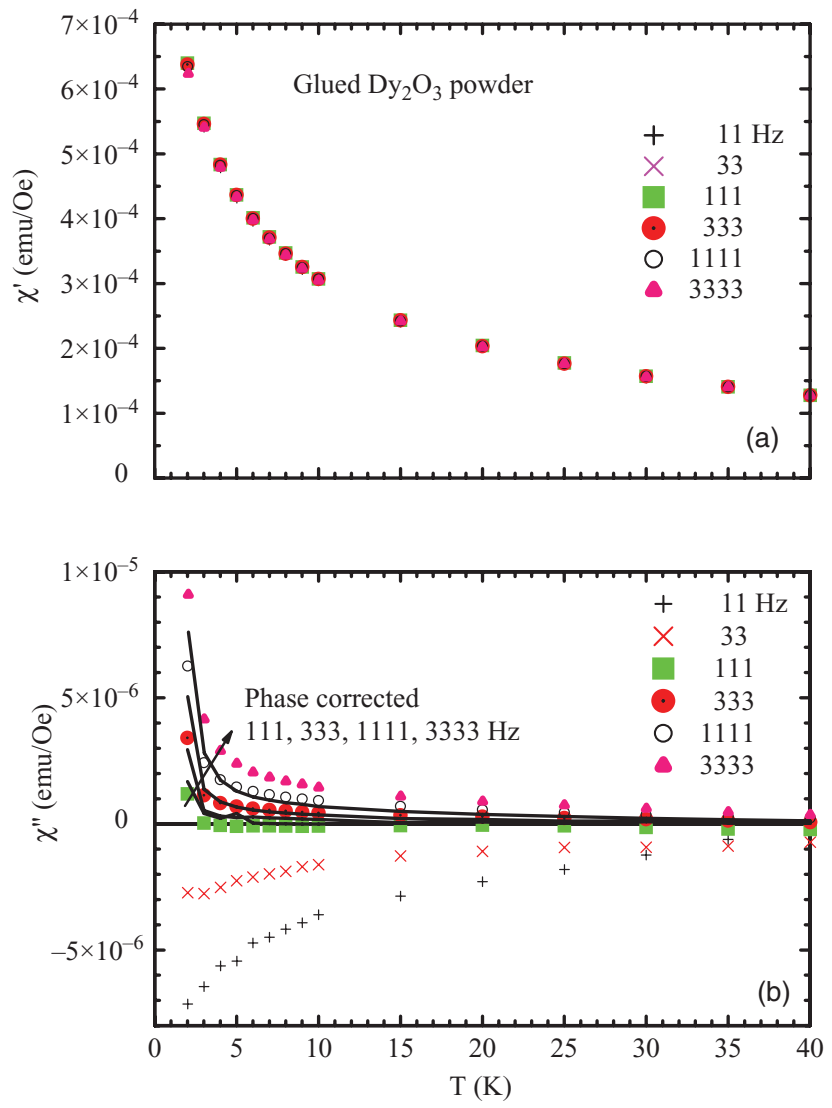

FIG. 1. (Color online) Real and imaginary ac moment susceptibilities, $\chi^{\prime}$ (a) and $\chi^{\prime \prime}(\mathrm{b})$, of the glued $\mathrm{Dy}_{2} \mathrm{O}_{3}$ standard sample measured at $H_{m}=10 \mathrm{Oe}$ and $f$ from 11 to $3333 \mathrm{~Hz}$ as a function of temperature $T$. The lines in (b) show the phase corrected $\chi^{\prime \prime}$ (see Fig. 9) with the arrow indicating the direction of increasing frequency.

where $\mu_{0}=4 \pi \times 10^{-7} \mathrm{H} / \mathrm{m}$ is the permeability of free space, $m_{0}\left(\mathrm{Am}^{2}\right)$ and $n$ are the elementary moment of the material and its number per unit volume, $k_{B}=1.381 \times 10^{-23} \mathrm{~J} / \mathrm{K}$ is the Boltzmann constant, and $T_{0}(\mathrm{~K})$ is the Weiss constant. Since all these quantities and the volume are constant for the sample, Eq. (1) may be conveniently written for the moment susceptibility $\chi_{\mathrm{CW}}$ in electromagnetic units as

$$
1 / \chi_{\mathrm{CW}}=\left(T-T_{0}\right) / C,
$$

where $C$ is a constant. For comparing experimental data with Eq. (2), we calculate the magnitude and phase of the measured $\chi$ as

$$
\begin{aligned}
|\chi| & =\sqrt{\chi^{\prime 2}+\chi^{\prime \prime 2}}, \\
\phi & =\arctan \left(\chi^{\prime \prime} / \chi^{\prime}\right),
\end{aligned}
$$

and plot $1 /|\chi|$ as a function of $T$ in Fig. 2(a) for $f=333$ and $1111 \mathrm{~Hz}$, at which $\chi$ measurements may be performed with higher resolution and smaller $T$-dependent magnitude error. ${ }^{5}$ The straight line shows the best Curie-Weiss fit to the data, with $C^{-1}=138.6 \mathrm{Oe} \mathrm{emu}^{-1} \mathrm{~K}^{-1}$ and $T_{0}=-16.8 \mathrm{~K}$. In order to show the relative difference between the data points of $1 /|\chi|$ and the line of $1 / \chi_{\mathrm{CW}}$, the scale of $1 /|\chi|$ is converted to logarithmic in Figs. 2(b) and 2(c) for $T$ from 34 to $110 \mathrm{~K}$ and from 110 to $310 \mathrm{~K}$, respectively. We see that the measured $1 /|\chi|$ is accurately $f$ independent between 35 and $150 \mathrm{~K}$ and 

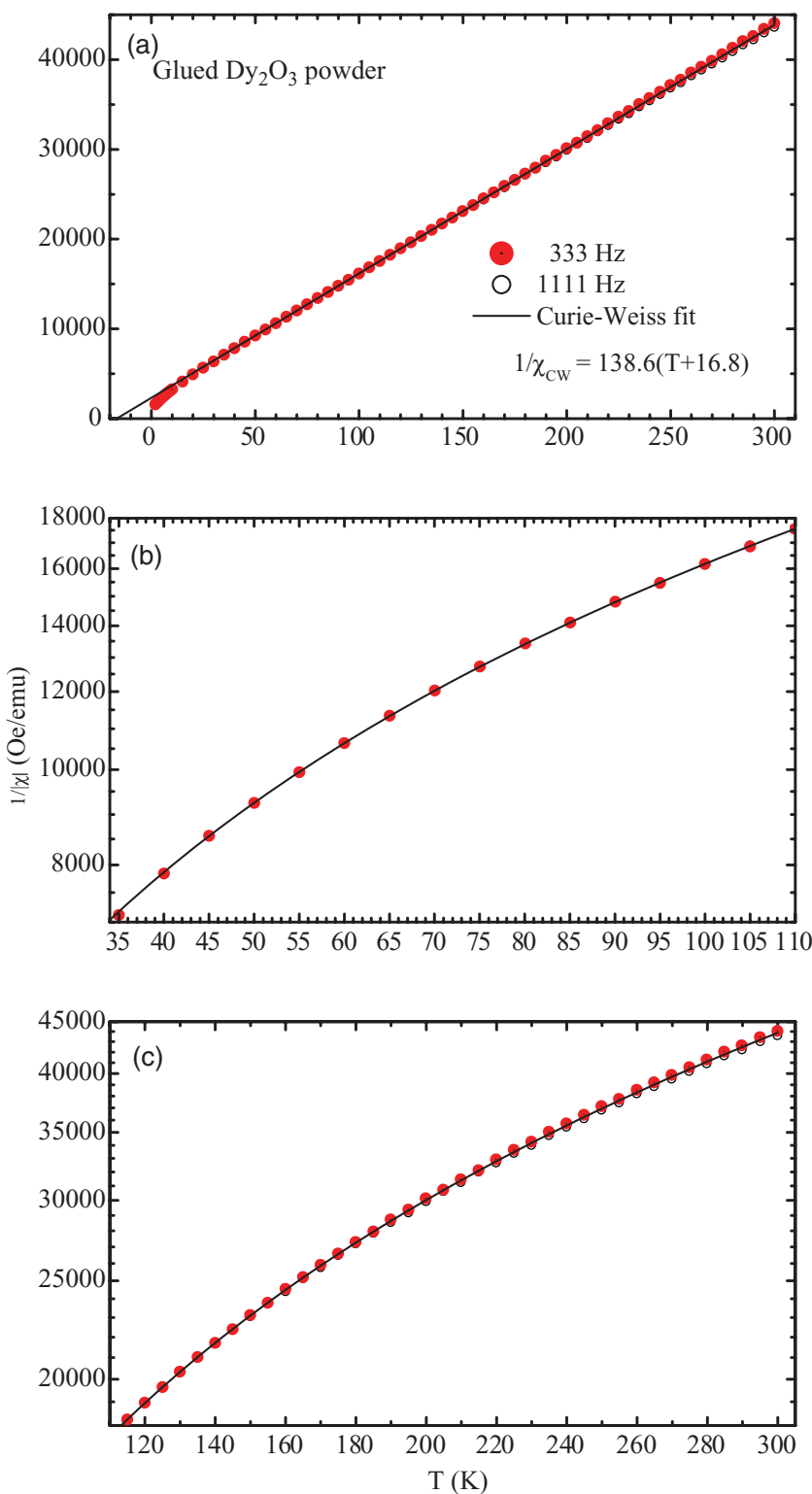

FIG. 2. (Color online) $1 /|\chi|$ of the glued $\mathrm{Dy}_{2} \mathrm{O}_{3}$ sample at $f=333$ and $1111 \mathrm{~Hz}$ (symbols) and $1 / \chi_{\mathrm{CW}}$ (line) as functions of $T$. Linear vertical scale is used in (a), so that the Curie-Weiss relation is linear, but logarithmic vertical scale is used in (b) and (c), in order to show clearly the relative differences among the data at each value of $T$.

it may have about $1 \%$ difference at $300 \mathrm{~K}$ between $f=333$ and $1111 \mathrm{~Hz}$. The fitting $1 / \chi_{\mathrm{CW}}$ line is located in between with a difference about $0.1 \%$ from the average $1 /|\chi|$ at both values of frequency when $T \geq 45 \mathrm{~K}$, but the line is above the data points for $0.6 \%$ and $1 \%$ at $T=40$ and $35 \mathrm{~K}$, respectively. The fitting error is remarkably increased at even lower $T$, as seen from Fig. 2(a).

Thus, the Curie-Weiss moment susceptibility of the particular glued $\mathrm{Dy}_{2} \mathrm{O}_{3}$ sample is finally obtained as

$$
\chi_{\mathrm{CW}}=[138.6(T+16.8)]^{-1}[\mathrm{emu} / \mathrm{Oe}],
$$

which can be used for low-field calibration of magnetometers at $T>45 \mathrm{~K}$.
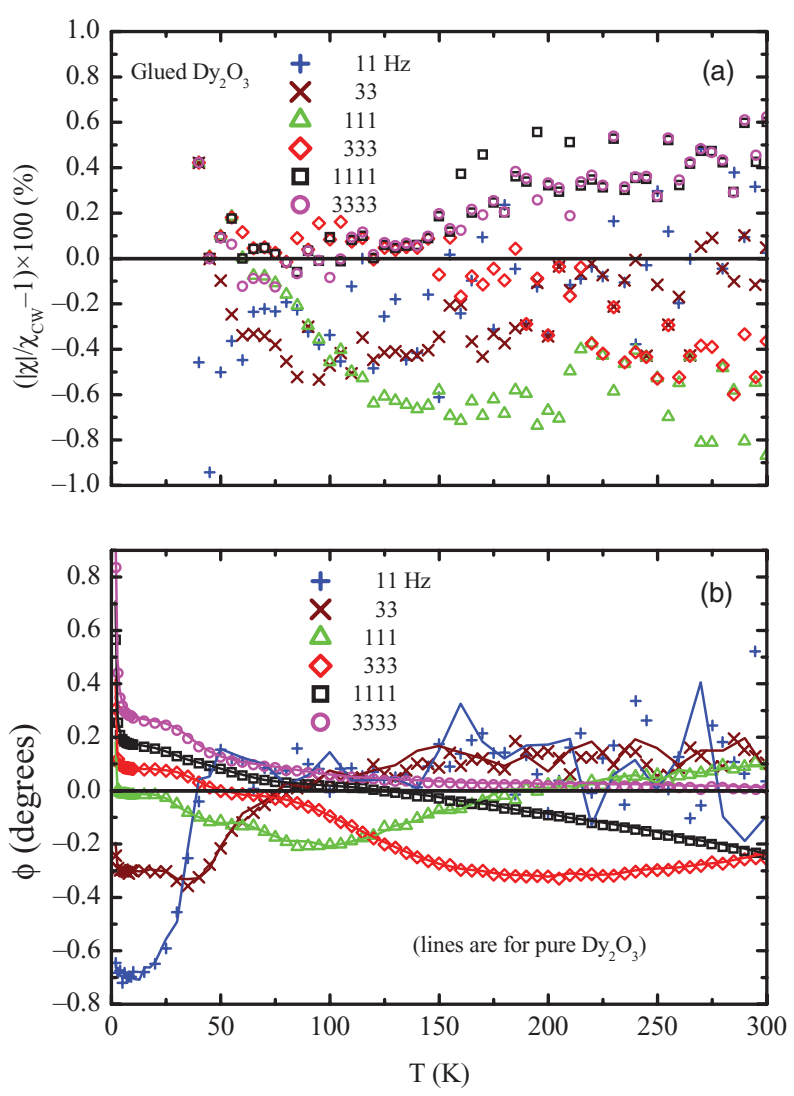

FIG. 3. (Color online) (a) Relative magnitude error $\left(|\chi| / \chi_{\mathrm{CW}}-1\right) \times 100$ (\%) at $T>39 \mathrm{~K}$ and (b) phase error of ac susceptibility measurements at frequencies between 11 and $3333 \mathrm{~Hz}$, determined by measuring the glued $\mathrm{Dy}_{2} \mathrm{O}_{3}$ sample, as functions of $T$ (symbols). The phase error determined by measuring a pure $\mathrm{Dy}_{2} \mathrm{O}_{3}$ sample is also shown in (b) by lines.

\section{B. Calibration of ac susceptometer at $\boldsymbol{H}_{\mathrm{dc}}=\mathbf{0}$}

Using $\chi_{\mathrm{CW}}$ in Eq. (4) as a standard, we calculate the relative error of the measured $|\chi|,|\chi| / \chi_{\mathrm{CW}}-1$, as a function of frequency and temperature, as shown in Fig. 3(a). This error is between $-0.8 \%$ and $0.6 \%$ at all values of frequency and temperature, which is consistent with $\pm 0.7 \%$ calibrated by the copper cylinder stated in Ref. 5. Since the standard $\phi$ is zero for paramagnetism at audio frequencies, the error in $\phi$ is actually the measured $\phi$ itself, as shown in Fig. 3(b). It is within $\pm 0.3^{\circ}$ and also comparable with the same error at $f=111-1111$ stated in Ref. 5. However, there are anomalies occurring at $T<10 \mathrm{~K}$, where $\phi$ rises sharply.

\section{Calibration of SQUID magnetometer}

The glued $\mathrm{Dy}_{2} \mathrm{O}_{3}$ sample was measured by the SQUID magnetometer at stepwise changed field from $-70 \mathrm{kOe}$ to $70 \mathrm{kOe}$ to $-70 \mathrm{kOe}$ at $T=10,20,50,100,200$, and $300 \mathrm{~K}$ after centering at each temperature. The field was set in noovershoot mode. The magnetic moment at each field was measured with one scan of distance $4 \mathrm{~cm}$ and 64 points $^{2}$ applying the "iterative regression" algorithm incorporated in the software. The SQUID magnetometer had been calibrated independently by using a Pd standard sample but a data correction will be made later in Sec. VI after the error due to sample dimensions is analyzed. 

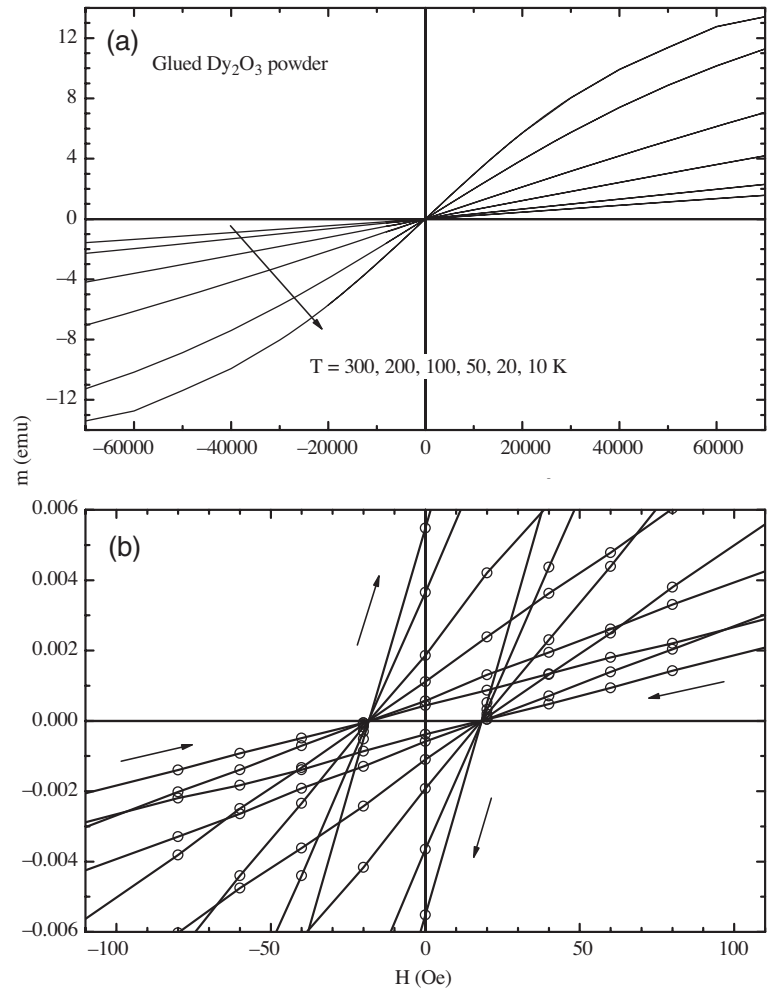

FIG. 4. Hysteresis $m$ vs $H$ curves of the glued $\mathrm{Dy}_{2} \mathrm{O}_{3}$ sample measured by the dc SQUID magnetometer. The arrow in (a) indicates the direction of decreasing $T$. The arrows in (b) indicate the sense of state evolution.

The results are shown in Fig. 4(a) for the complete field range and in Fig. 4(b) for a low field portion, where hysteresis can be seen. Arrows in Fig. 4(b) indicate the directions of magnetic state evolution, which has an opposite sense to that of a normal hysteresis loop.

The hysteresis is a consequence of the error in field produced by superconducting magnet. The instrument reported field is proportional to the current flowing in the superconducting solenoid calculated from the Ampère law but the actual applied field at the sample is the sum of this field produced by the current and the field produced by the magnetic poles in the magnetized superconductor itself. According to the critical-state model, ${ }^{7-9}$ a positive magnetic moment is induced in a hard superconductor and is proportional to the critical-current density $J_{c}$, when the field decreases from a large positive value. Such a moment will produce a negative demagnetizing field in the bore of the magnet. When the field increases from a large negative value, the moment becomes negative and the demagnetizing field becomes positive. This is the reason for the hysteresis shown in Fig. 4(b), as already explained in Ref. 3. Since $J_{c}$ decreases with increasing the magnitude of the field $|H|,{ }^{8,9}$ this error decreases with increasing $|H|$. However, we may add $+/-18.2$ Oe to the reported field values on the ascending/descending curve to effectively reduce the relative error in the entire range, since a major portion of the error is corrected at low $|H|$ and the over correction is much smaller than the field value at high $|H|$. This will be further discussed when Fig. 12 is shown. After such a correction, the comparison between the $m / H$ measured by the SQUID magnetometer and

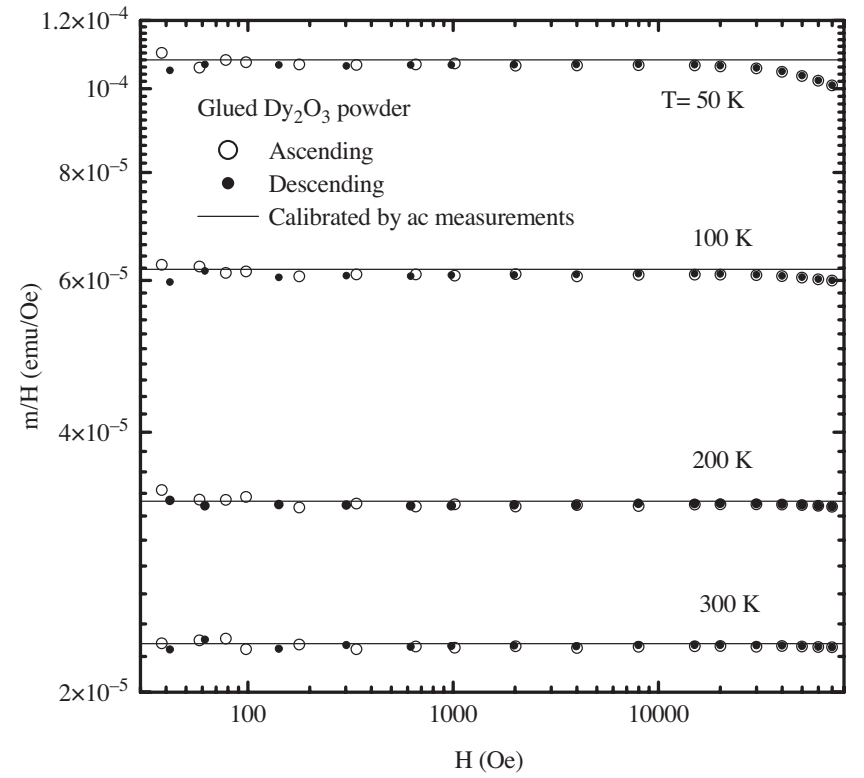

FIG. 5. The $m / H$ calculated from the $m$ vs $H$ curves measured by the dc SQUID magnetometer at $T=50,100,200$, and $300 \mathrm{~K}$ after a field shift of \pm 18.2 Oe compared with the ac moment susceptibility of the glued $\mathrm{Dy}_{2} \mathrm{O}_{3}$ sample obtained by calibration of the ac susceptometer, Eq. (4).

$\chi_{\mathrm{CW}}$ calculated from Eq. (4) is shown in Fig. 5. We see that for $T=50,100,200$, and $300 \mathrm{~K}$, the measured ascending and descending $m / H$ are quite stable and lower than $\chi_{\mathrm{CW}}$ of Eq. (4) by about $1 \%$ in a large field interval. Thus, the SQUID magnetometer can be calibrated by using the $\mathrm{Dy}_{2} \mathrm{O}_{3}$ sample, which has 1 order of magnitude larger $\chi$ than the Pd sample so a higher resolution at low fields can be achieved.

\section{HIGH-FIELD CALIBRATION}

\section{A. Field and temperature dependence of magnetic moment}

The measured $m$ vs $H$ curves after the field correction (i.e., the shift of $+/-18.2$ Oe) are shown in Fig. 6. All the experimental curves at different $T$ may be well fitted by the classical theory of paramagnetism in terms of the Langevin function, plotted by the red solid lines. In this theory, ${ }^{6}$ the magnetization of a paramagnetic material is expressed, after combining with the Curie-Weiss law, by

$$
M=M_{0} L(x),
$$

where $M_{0}=n m_{0}$ and the Langevin function

$$
L(x)=\operatorname{coth}(x)-1 / x
$$

with $x$ expressed in SI units as

$$
x=\frac{\mu_{0} m_{0} H}{k_{B}\left(T-T_{0}\right)} .
$$

It can be derived from Eqs. (5)-(7) that the differential susceptibility at any values of $H$ and $T$ is

$$
\frac{d M}{d H}=\left(1-\operatorname{coth}^{2} x+\frac{1}{x^{2}}\right) \frac{n \mu_{0} m_{0}^{2}}{k_{B}\left(T-T_{0}\right)},
$$

whose limit at $H=0$ is Eq. (1). 
To compare with the measured data in electromagnetic units, these equations are rewritten as

$$
\begin{gathered}
m=\frac{3 k_{B} C}{m_{0}} L(x), \\
\frac{d m}{d H}=\left(1-\operatorname{coth}^{2} x+\frac{1}{x^{2}}\right) \frac{3 C}{\left(T-T_{0}\right)},
\end{gathered}
$$

where

$$
x=\frac{m_{0} H}{k_{B}\left(T-T_{0}\right)} .
$$

As Eq. (4), all these equations are valid for the data at $T$ $>45 \mathrm{~K}$, where the only adjustable parameter is $m_{0} / k_{B}$, which should be obtained by fitting the measured $m(H)$ curves. However, the measured $m(H)$ curves at $T \geq 100 \mathrm{~K}$ are basically linear, for which Eq. (9) is reduced to Eq. (2), where $m_{0} / k_{B}$ is absent. Thus, we use data for $T=20 \mathrm{~K}$, where nonlinear $m$ vs $H$ is clearly shown, to start the fit. Unfortunately, the Curie-Weiss law is not valid accurately for $T=20 \mathrm{~K}$, and we choose to assume the same $C$ in Eq. (2) and to change $T_{0}$ to an effective one $-15.5 \mathrm{~K}$ to get the value of $\chi$ measured by ac susceptibility at $20 \mathrm{~K}$. The obtained fitting parameter is $m_{0} / k_{B}=9.8 \times 10^{-4} \mathrm{~K} / \mathrm{Oe}$. With these parameter, the results for $\geq 50 \mathrm{~K}$ are well fitted, as seen in Fig. 6. Even so, the results for $T=10 \mathrm{~K}$ cannot be well fitted by such a value of $m_{0} / k_{B}$ after the effective $T_{0}$ is properly set as $-13.5 \mathrm{~K}$; a good fit requires $m_{0} / k_{B}=1.1 \times 10^{-3} \mathrm{~K} / \mathrm{Oe}$ at $T=10 \mathrm{~K}$.

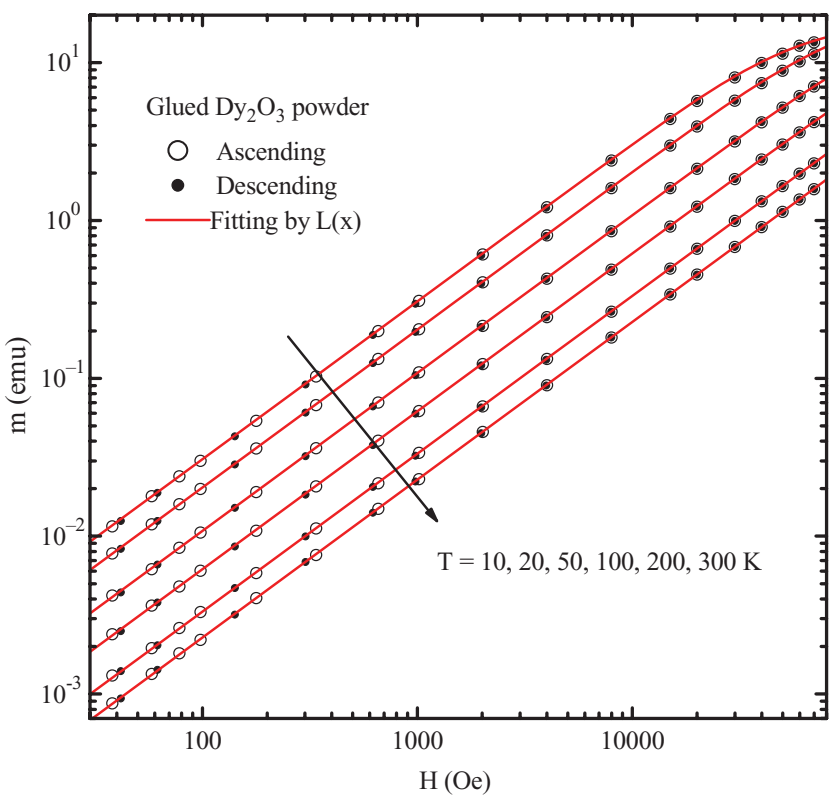

FIG. 6. (Color online) $m$ vs $H$ loops of the glued $\mathrm{Dy}_{2} \mathrm{O}_{3}$ sample measured by the dc SQUID magnetometer at $T=10,20,50,100,200$, and $300 \mathrm{~K}$, increasing in the arrow direction, after a field shift of \pm 18.2 Oe. Open and solid circles show the ascending and descending branches, respectively. Red solid lines show the Curie-Weiss and Langevin function fitting.

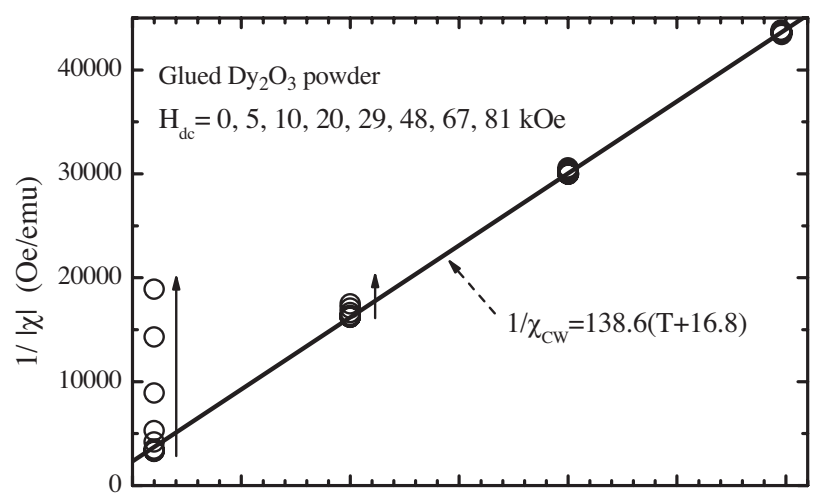

(a)

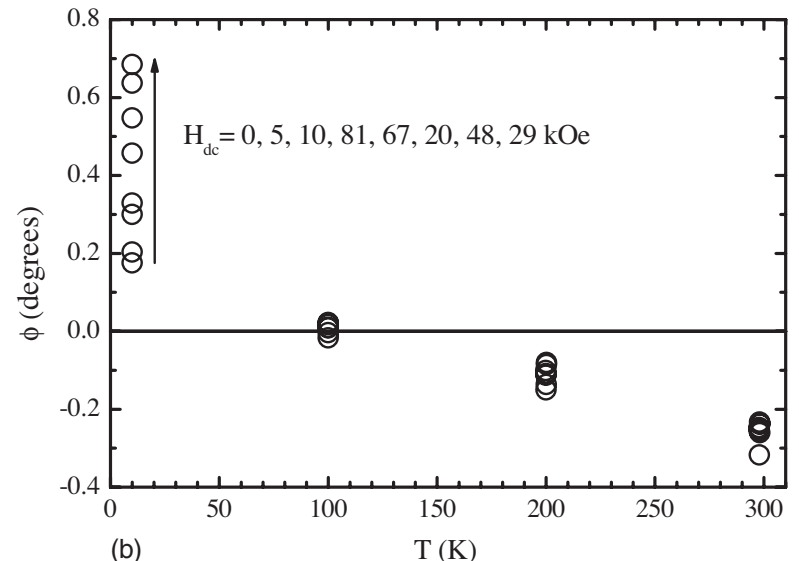

FIG. 7. $1 /|\chi|$ (a) and $\phi$ (b) of the incremental ac moment susceptibility of the glued $\mathrm{Dy}_{2} \mathrm{O}_{3}$ sample measured by the ac susceptometer at $H_{\mathrm{dc}}$ from 0 to $81 \mathrm{kOe}$ and $T=10,100,200$, and $298 \mathrm{~K}$. The arrows in (a) indicate the direction of increasing $H_{\mathrm{dc}}$. The arrow in (b) indicates the change of $H_{\mathrm{dc}}$ from 0 to $29 \mathrm{kOe}$ as listed.

\section{B. Calibration of incremental $\chi$}

After the $m(H)$ fits by Eqs. (9) and (11), Eqs. (10) and (11) may be used for the calibration of incremental $\chi$ at different values of $H_{\mathrm{dc}}, f$, and $T$.

The $1 /|\chi|$ and $\phi$ of the incremental ac susceptibility measured at $H_{m}=10 \mathrm{Oe}, f=1111 \mathrm{~Hz}$, eight values of $H_{\mathrm{dc}}$ between 0 and $80 \mathrm{kOe}$, and $T=10,100,200$, and $298 \mathrm{~K}$ are shown in Figs. 7(a) and 7(b), respectively. We see in Figs. 7(a) and $7(\mathrm{~b})$ that $1 /|\chi|$ increases monotonically and $\phi$ increases and then decreases with increasing $H_{\mathrm{dc}}$ at $T=10 \mathrm{~K}$; similar but smaller variation occurs at $T=100 \mathrm{~K}$ for $1 /|\chi|$ but not for $\phi$. The behavior at $T=20 \mathrm{~K}$ is similar to $T=10 \mathrm{~K}$ but less pronounced (data not shown).

We plot the measured $\chi^{\prime}$ and $\chi^{\prime \prime}$ in Figs. 8(a) and 8(b), respectively, as functions of $H_{\mathrm{dc}}$ at $T=10$ and $100 \mathrm{~K}$. We see that $\chi^{\prime}$ decreases with increasing $H_{\mathrm{dc}}$ at both values of $T$, and $\chi^{\prime \prime}$ shows a peak at $T=10 \mathrm{~K}$ but equals zero for $T$ $=100 \mathrm{~K}$. Regarding $\chi^{\prime}$ as $d m / d H$ at $H=H_{\mathrm{dc}}$, we compare it with the results calculated from Eqs. (10) and (11), as plotted by red lines in Fig. 8(a). We see that the line matches well the data points for $T=100 \mathrm{~K}$, but the line is obviously above the points at $T=10 \mathrm{~K}$. It seems that the properties of the $\mathrm{Dy}_{2} \mathrm{O}_{3}$ are anomalous at $T=10 \mathrm{~K}$ for paramagnetism, and the over-low incremental $\chi^{\prime}$ is accompanied by the $\chi^{\prime \prime}$ peak. 

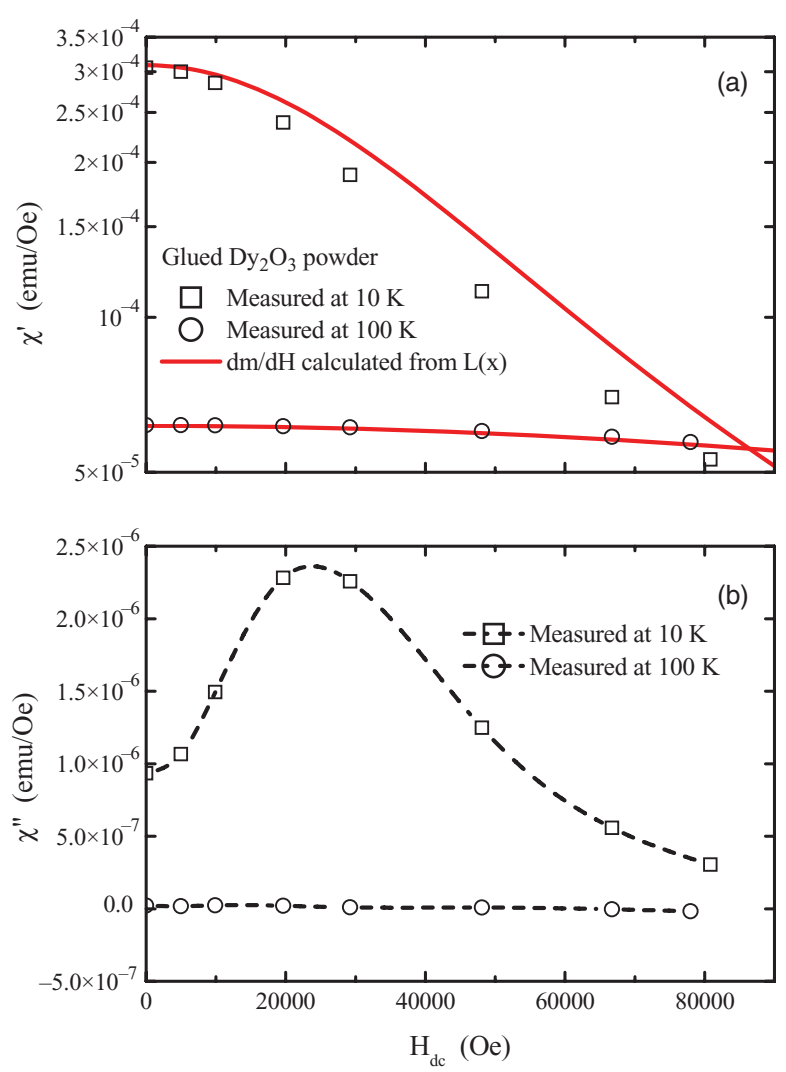

FIG. 8. (Color online) Real and imaginary incremental ac moment susceptibilities, $\chi^{\prime}$ (a) and $\chi^{\prime \prime}$ (b), of the glued $\mathrm{Dy}_{2} \mathrm{O}_{3}$ sample measured at 10 and $100 \mathrm{~K}$ as a function of $H_{\mathrm{dc}}$. In (a), red solid lines show results of differential susceptibilities calculated from Eqs. (10) and (11).

\section{ANOMALIES}

\section{A. Low- $T$ anomalies in susceptibility}

Anomalies in phase similar to those shown in Fig. 3(b) are not reported in Ref. 5, where a high-quality superconducting film is measured at a low field in the Meissner state so that $\phi$ should be 0 . Comparing the measured $\phi$ vs $T$ of the superconductor and $\mathrm{Dy}_{2} \mathrm{O}_{3}$ in Fig. 9, we see that the $\phi$ of the former is stable at $T<20 \mathrm{~K}$, but for the latter, it is larger than the former and increases quickly with decreasing $T$ to below $5 \mathrm{~K}$. This suggests that the low- $T$ anomalous $\phi$ in Fig. 3(b) should not be a consequence of the error in ac susceptibility measurements alone, but be also from a possible low- $T$ phase transition of $\mathrm{Dy}_{2} \mathrm{O}_{3}$ itself. Using the results of the superconducting film as a standard, the phase corrected $\chi^{\prime \prime}$ as a function of $T$ is plotted in Fig. 1(b), showing $\chi^{\prime \prime}>0$ till $T=40 \mathrm{~K}$ with a systematic $f$ dependence.

Such an anomaly may be related to the over-low incremental $\chi^{\prime}$ and the $\chi^{\prime \prime}$ peak occurring at low $T$.

It is worth mentioning the early work ${ }^{10}$ reporting that $\mathrm{Dy}_{2} \mathrm{O}_{3}$ undergoes a transition into antiferromagnetic state below the Neel temperature $T_{N}=1.2 \mathrm{~K}$. This temperature is much smaller in magnitude than the Weiss constant $T_{0}$ $=-16.8 \mathrm{~K}$ obtained in the present work, indicating a high degree of frustration between the magnetic moments in the ordered state. ${ }^{11}$ The much higher Weiss constant $T_{0}$ $=-3.2 \mathrm{~K}$ quoted in Ref. 10 is probably due to the very lim-

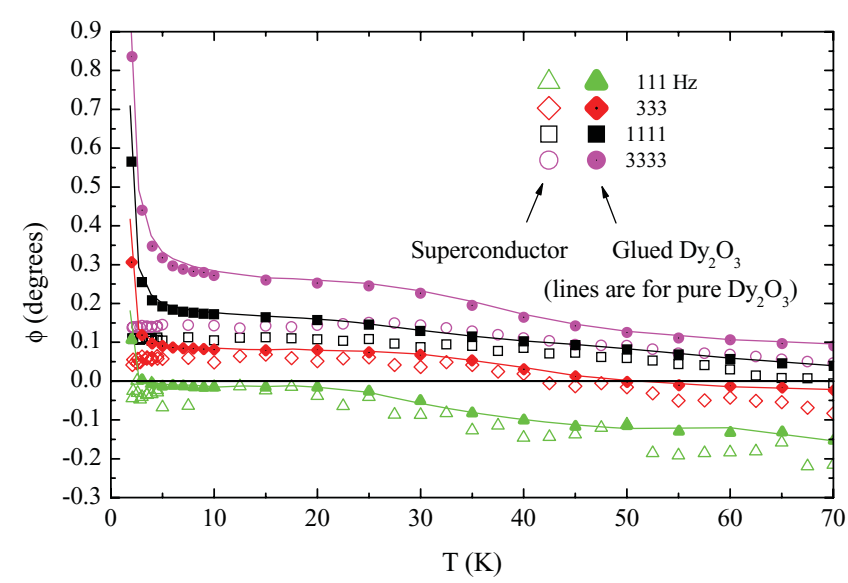

FIG. 9. (Color online) Comparison of the phase of the ac susceptibility of the glued (solid symbols) and a pure (lines) $\mathrm{Dy}_{2} \mathrm{O}_{3}$ samples with a superconducting film in the Meissner state studied in Ref. 5 (open symbols).

ited temperature range (up to only $4.2 \mathrm{~K}$ ) from where the data were extrapolated. A phase transition of $\mathrm{Dy}_{2} \mathrm{O}_{3}$ at $T \approx 1 \mathrm{~K}$ was also mentioned in Ref. 12.

It is important to realize that the true Curie-Weiss behavior sets in well above $T_{N}$, when any short-range order effects would have died out and all the levels of the crystal-field-split ground multiplet of $\mathrm{Dy}^{3+}$ ion are significantly populated. ${ }^{13}$ We argue that this is valid at temperatures above $45 \mathrm{~K}$, where a good fit of the experimental data is found.

\section{B. Over-large $m_{0}$ at low fields}

According to the quantum mechanical theory of paramagnetism, the Langevin function $L(x)$ should be replaced by the Brillouin function $B_{J}(y)$ as ${ }^{6}$

$$
M=M_{0} B_{J}(y),
$$

where

$$
M_{0}=n g \mu_{B} J
$$

and the Brillouin function is

$$
B_{J}(y)=\frac{2 J+1}{2 J} \operatorname{coth}\left(\frac{2 J+1}{2 J} y\right)-\frac{1}{2 J} \operatorname{coth}\left(\frac{y}{2 J}\right)
$$

with $y$ expressed in SI units as

$$
y=\frac{\mu_{0} g \mu_{B} J H}{k_{B}\left(T-T_{0}\right)} .
$$

The corresponding low-field susceptibility may be derived as

$$
\left.\frac{d M}{d H}\right|_{H \rightarrow 0}=\frac{n \mu_{0} g^{2} \mu_{B}^{2} J(J+1)}{3 k_{B}\left(T-T_{0}\right)}=\frac{n \mu_{0} m_{0}^{2}}{3 k_{B}\left(T-T_{0}\right)} .
$$

Since the elementary moment of $\mathrm{Dy}_{2} \mathrm{O}_{3}$ is carried by $\mathrm{Dy}^{3+}$, whose spin, orbital, and total angular momenta are $S=5 / 2$, $L=5$, and $J=15 / 2$, so that the $g$ factor

$$
g=1+\frac{J(J+1)+S(S+1)-L(L+1)}{2 J(J+1)}=4 / 3 .
$$

The elementary moment $m_{0}=g \mu_{B} \sqrt{J(J+1)}$ $=10.6 \mu_{B}$ may be calculated from Eqs. (16) and (17). 
Since $m_{0} / k_{B}$ is fixed in this case, the measured $m(H)$ curve cannot be well fitted. It is interesting to note that the classical theory fits lead to $m_{0}=14.6 \mu_{B}$ and $m_{0}=16.4 \mu_{B}$ for $T \geq 20 \mathrm{~K}$ and $T=10 \mathrm{~K}$, respectively.

This $T$-dependent over-large $m_{0}$ detected from the glued $\mathrm{Dy}_{2} \mathrm{O}_{3}$ powder is an anomalous behavior with respect to paramagnetism. However, we have validated Eq. (16) with $m_{0}=g \mu_{B} \sqrt{J(J+1)}=10.6 \mu_{B}$ by measuring a pure $\mathrm{Dy}_{2} \mathrm{O}_{3}$ powder sample of known mass, as described below. Therefore, the discovered over-large $m_{0}$ at low fields is actually a consequence of over-small saturation $M_{0}$. A value of $M_{0}=10 n \mu_{B}$ is calculated from Eqs. (13) and (17) for $\mathrm{Dy}^{3+}$, but $M_{0} \approx 5.3 n \mu_{B}$ is obtained from the magnetization of the $\mathrm{Dy}_{2} \mathrm{O}_{3}$ powder sample at $T=1.8 \mathrm{~K}$ and $\mu_{0} H=7 \mathrm{~T}$. We note that even smaller $M_{0} \approx 2.8 n \mu_{B}$ was reported previously for $\mathrm{Dy}_{2} \mathrm{O}_{3}$ powder. ${ }^{14}$ Since the actual magnetic saturation is lower than that appearing in the quantum mechanical theory of paramagnetism, the measured $m(H)$ curves cannot be well fitted by this theory, but they still can be well fitted by the classical theory of paramagnetism, if $m_{0}$ may be adjusted arbitrarily. The over-large values of $m_{0}$ are thus obtained by Langevin fitting to the measured $m(H)$ curves.

\section{PURE $\mathrm{DY}_{2} \mathrm{O}_{3}$ POWDER AS A PRIMARY STANDARD}

Regarding $n$ as the number of $\mathrm{Dy}^{3+}$ per unit mass, calculated by the molar mass $373.0 \mathrm{~g} / \mathrm{mol}$ of $\mathrm{Dy}_{2} \mathrm{O}_{3}$ and the Avogadro constant $N_{A}=6.022 \times 10^{23} \mathrm{~mol}^{-1}$, and assuming $m_{0}=10.6 \mu_{B}$, the Curie-Weiss susceptibility per unit mass, written as $\chi_{m, \mathrm{CW}}$, may be calculated from Eq. (1) in both unit systems as

$$
\begin{aligned}
\chi_{m, \mathrm{CW}} & =\left[13.28\left(T-T_{0}\right)\right]^{-1}[\mathrm{emu} / \mathrm{Oe} / \mathrm{g}] \\
& =\left[1.056\left(T-T_{0}\right)\right]^{-1} \times 10^{-6}\left[\mathrm{~m}^{3} / \mathrm{kg}\right] .
\end{aligned}
$$

There is a $T$-independent diamagnetic contribution from the core electrons of the atoms. From the Pascal constant of $\mathrm{Dy}^{3+}$ and $\mathrm{O}^{-2}$ to be $-19 \times 10^{-6}$ and $-12 \times 10^{-6} \mathrm{emu} / \mathrm{mol},{ }^{15}$ the diamagnetic partial susceptibility of $\mathrm{Dy}_{2} \mathrm{O}_{3}$ is calculated to be $-2 \times 10^{-7} \mathrm{emu} / \mathrm{Oe} / \mathrm{g}$, which is $10^{3}$ times in magnitude smaller than $\chi_{m, \mathrm{CW}}$ at $300 \mathrm{~K}$ and so negligible at $T$ $<300 \mathrm{~K}$. Thus, pure $\mathrm{Dy}_{2} \mathrm{O}_{3}$ powder with known mass can be used as a primary standard if Eq. (18) is valid.

The validity of Eq. (18) derived from quantum mechanical theory is tested experimentally. We have prepared a spherical sample of $\mathrm{Dy}_{2} \mathrm{O}_{3}$ powder of purity $99.9 \%$ (Alfa Aesar, Germany, provided by Quantum Design) covered with a small piece of food wrap foil to avoid extra diamagnetic contribution, and measured its $m$ vs $T$ by the SQUID magnetometer at $H=1000 \mathrm{Oe}$. In order to get accurate $H$, the superconducting magnet was quenched before measurements. The sample was automatically centered at each value of $T$. The results are given in Fig. 10, from which we see that the measured data may be well fitted by

$$
\chi_{m}=[13.30(T+17.5)]^{-1}[\mathrm{emu} / \mathrm{Oe} / \mathrm{g}] .
$$

The constant factor is practically the same as that in Eq. (18), but $T_{0}=-17.5 \mathrm{~K}$ is $0.7 \mathrm{~K}$ lower than that for the glued sample determined by ac measurements. We suspect that the dif-
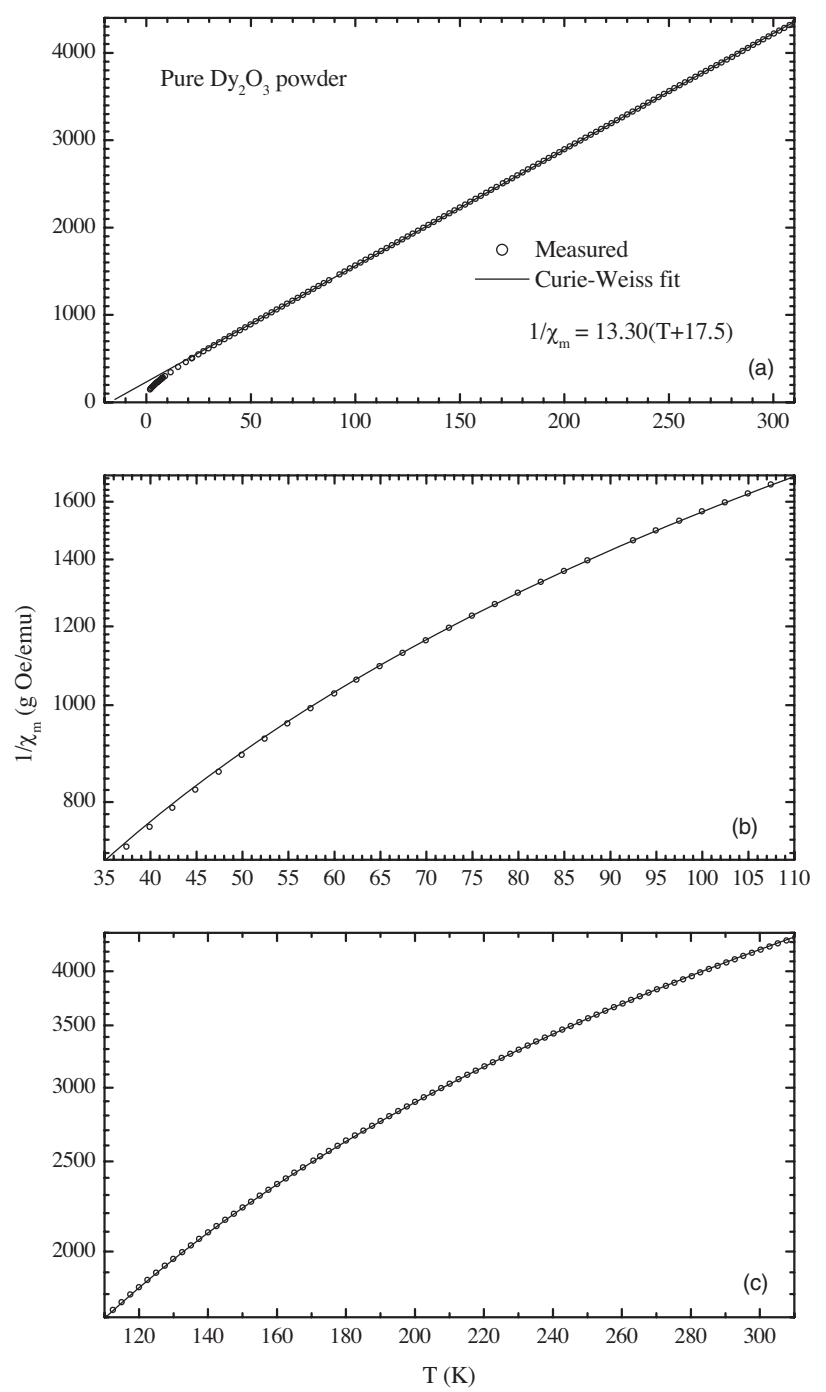

FIG. 10. $1 / \chi_{m}$ of a pure $\mathrm{Dy}_{2} \mathrm{O}_{3}$ powder sample measured by the dc SQUID magnetometer at $H=1000 \mathrm{Oe}$ as a function of $T$ and its Curie-Weiss fit. Linear vertical scale is used in (a), so that the Curie-Weiss relation is linear, but logarithmic vertical scale is used in (b) and (c), in order to show clearly the relative differences among the data at each value of $T$.

ference in $T_{0}$ comes from different errors in $T$ between the ac and dc magnetometers, since $T_{0}=-17.5 \mathrm{~K}$ is also obtained by fitting the data in Fig. 5 for the glued sample and $T_{0}=-16.9 \mathrm{~K}$ has been obtained by ac measurements of another pure powder sample. Without direct calibration of $T$ for the magnetometers, $T_{0}=-17 \mathrm{~K}$ may be chosen in Eq. (18) for simplicity.

We should mention that previous measurements in Ref. 16 gave $m_{0}=9.14 \mu_{B}$ for $\mathrm{Dy}^{3+}$, which is $14 \%$ smaller than $10.6 \mu_{B}$.

The equal validity of the glued and pure $\mathrm{Dy}_{2} \mathrm{O}_{3}$ powder samples in the phase calibration of the ac susceptometer may be seen from Figs. 3(b) and 9, where symbols and lines are the results of the glued and pure samples, respectively.

\section{ERROR DUE TO FINITE SAMPLE DIMENSIONS}

Until now, we have overlooked an error in moment measurements due to finite sample dimensions. The moment 


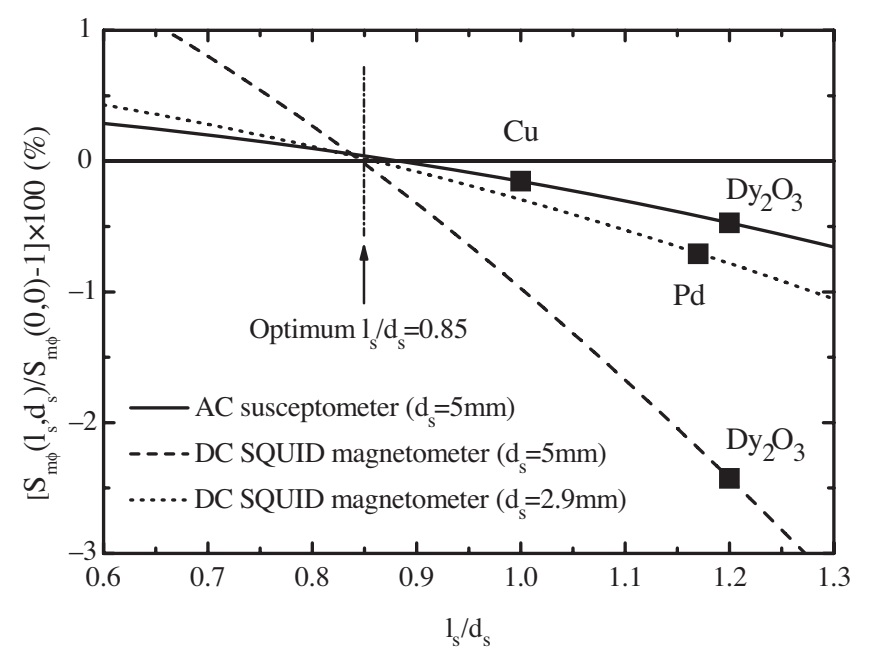

FIG. 11. The relative difference between cylindrical samples of length $l_{s}$ and diameter $d_{s}$ and a point moment in moment sensitivity with respect to the sensed flux linkage, $S_{m \phi}\left(l_{s}, d_{s}\right) / S_{m \phi}(0,0)-1$, as a function of $l_{s} / d_{s}$. The one-turn coil diameter is $19.4 \mathrm{~mm}$ for the dc SQUID magnetometer, and the coil diameter and length are 9 and $19 \mathrm{~mm}$, respectively, for the ac susceptometer. The sample diameter and length are 5 and $5 \mathrm{~mm}$ for copper, 5 and $6 \mathrm{~mm}$ for the glued $\mathrm{Dy}_{2} \mathrm{O}_{3}$, and 2.9 and $3.4 \mathrm{~mm}$ for $\mathrm{Pd}$.

sensitivity of magnetometers is defined ideally for a sample of negligible dimensions compared with the size of sensing coil or for a spherical sample of finite diameter. The calibration samples provided by Quantum Design are cylinders and their moment sensitivity should depend on their dimensions.

Since a uniformly magnetized cylinder is equivalent to a current-flowing uniformly wound thin solenoid, ${ }^{17}$ the flux linkage in the sensing coil produced by the magnetic moment of the sample can be calculated numerically based on the formula for the mutual inductance $L_{m}$ between two coaxial coils of radius $\rho_{1}$ and $\rho_{2}$ and distance $\delta,{ }^{18}$

$$
L_{m}=\mu_{0} \sqrt{\rho_{1} \rho_{2}}\left[\left(k_{m}-2 / k_{m}\right) K\left(k_{m}\right)+2 E\left(k_{m}\right) / k_{m}\right],
$$

where

$$
k_{m}^{2}=4 \rho_{1} \rho_{2}\left[\left(\rho_{1}+\rho_{2}\right)^{2}+\delta^{2}\right]^{-1} .
$$

To estimate the error, we consider one sensing coil (thin solenoid) of diameter $9 \mathrm{~mm}$ and length $19 \mathrm{~mm}$ for the ac susceptometer and one single-turn coil of diameter $19.4 \mathrm{~mm}$ for the dc SQUID magnetometer. ${ }^{19}$ Assuming the sample diameter to be $d_{s}=5$ or $2.9 \mathrm{~mm}$ (corresponding to the copper and the glued $\mathrm{Dy}_{2} \mathrm{O}_{3}$ cylinders or the Pd cylinder), we calculate the moment sensitivity with respect to the sensed flux linkage for a cylinder sample of length $l_{s}$ and diameter $d_{s}, S_{m \phi}\left(l_{s}, d_{s}\right)$. The resulting $S_{m \phi}\left(l_{s}, d_{s}\right) / S_{m \phi}(0,0)-1$ as a function of $l_{s} / d_{s}$ is shown in Fig. 11.

We see from Fig. 11 that the error for the ac moment measurements of the $\mathrm{Cu}$ and the glued powder is $-0.2 \%$ and $-0.5 \%$, respectively, and the error for the dc moment measurements of the Pd and the glued powder is $-0.7 \%$ and $-2.4 \%$, respectively. Since the pure powder sample is spherical, such an error is zero for its measurements.

Before making additional error analysis, we should mention that a magnitude correction was already made for the data of ac measurements with a factor $k=1.081$ obtained by measuring the copper standard, but a similar correction was not made yet for the data of dc measurements with another factor $k=0.980$ obtained by measuring the Pd standard. Combining the $-0.7 \%$ error, this factor should be replaced by $k=0.987$.

Multiplying 0.987 to 13.30 in Eq. (19) results in 13.13, which is $1.1 \%$ lower than the theoretical 13.28 in Eq. (18). The over-high susceptibility of the powder sample may partially result from a negative error in $H$ about $-0.3 \%$, estimated by measuring the initial and hysteresis loop after quenching the magnet. Considering this, the measured susceptibility is $0.8 \%$ higher than its theoretical value.

The ac susceptibility of the glued $\mathrm{Dy}_{2} \mathrm{O}_{3}$ cylinder measured by the ac susceptometer, which was calibrated and corrected by measuring the copper cylinder, has a $-0.3 \%$ error owing finite dimensions of both cylinders, so that 138.6 in Eq. (4) should be corrected to 138.2. The dc susceptibility of the glued sample measured by the dc SQUID magnetometer before correction has a $0.3 \%$ error owing to the finite dimensions of the $\mathrm{Pd}$ and $\mathrm{Dy}_{2} \mathrm{O}_{3}$ cylinders and $k=0.980$. As a result, the low-field dc susceptibility becomes about $1.6 \%$ lower than those calculated from the corrected Eq. (4).

We have calculated the moment sensitivity with respect to a single coil to calculate the error for the SQUID magnetometer rather than that with respect to the coil assembly with iterative regression as calculated in Ref. 19. However, both results are similar; for a cylinder of length and diameter $5 \mathrm{~mm}$, the error is $-0.97 \%$ and $-1.04 \%$ for the former and the latter calculations, respectively.

From the results shown in Fig 11, we may conclude that when cylindrical samples are used for calibrating ac and dc magnetometers, their optimum $l_{s} / d_{s}=0.85$, at which the error due to finite dimensions is less than $0.1 \%$. Standard samples provided by the factory have $l_{s} / d_{s} \approx 1.2$, which may result in an error about $-0.5 \%$.

\section{FURTHER DISCUSSION}

The difference about $1.6 \%$ in low-field susceptibility of the glued sample between the ac and dc measurements could be due to temperature, whose accuracy is $1 \%$ according to the manuals. We make the following comparison in $m(H)$ between the results obtained by direct SQUID magnetometer measurements and those calculated from Eqs. (9) and (11). The difference $\Delta m(H)$ between the measured and calculated $m$ at $T=200 \mathrm{~K}$ is plotted in Fig. 12 by red open squares. We see that the difference between the ascending and descending values remains constant at $H<1000 \mathrm{Oe}$ and it decreases with further increasing $H$ and that the average $\Delta m$ increases in magnitude with increasing $H$ remarkably at $H>1000$ Oe. If $200 \mathrm{~K}$ is replaced by $202 \mathrm{~K}$ in the calculation, then such an increase becomes a small oscillation as shown by black open circles. The constant $\Delta m$ at $H<1000$ Oe indicates the applicability of the constant field shift used for correcting the field in quite a large range of field.

We have calibrated dc and/or ac magnetometers by using a copper cylinder, a high-temperature superconducting film, a glued $\mathrm{Dy}_{2} \mathrm{O}_{3}$ powder sample, and a pure $\mathrm{Dy}_{2} \mathrm{O}_{3}$ powder 


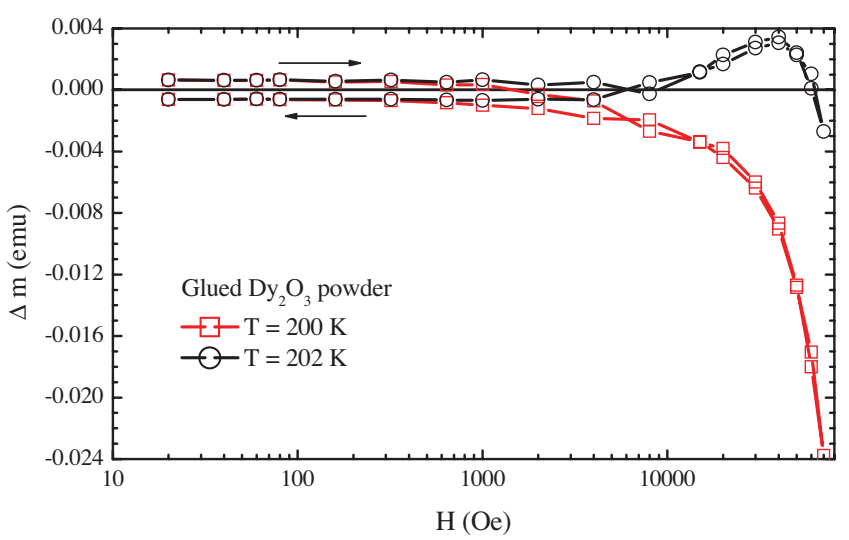

FIG. 12. (Color online) The difference between the $m$ vs $H$ loop of the glued sample directly measured by the dc SQUID magnetometer (without field shift) at $T=200 \mathrm{~K}$ and its Curie-Weiss and Langevin function fitting with $T=200$ and $202 \mathrm{~K}$.

sample. It is necessary to compare the different standards. Based on its ac susceptibility calculated from the eddy-current effects, the copper cylinder can calibrate ac susceptometers only. It may be regarded as a quasiprimary standard sample since its properties and the performance of the ac susceptometer can be mutually calibrated. ${ }^{5}$ The calibration may be performed in the entire $T, H_{m}$, and $f$ range, but owing to a remarkable magnetoresistance, the incremental susceptibility at $H_{\mathrm{dc}}>0$ cannot be calibrated by it. Based on the calculated susceptibility in the Meissner state, the superconducting film may also be regarded as a primary standard sample. It can be used at enough low temperatures and very low fields, satisfying the calibration of ac susceptometer at $T<70 \mathrm{~K}$. The glued $\mathrm{Dy}_{2} \mathrm{O}_{3}$ powder sample is not a primary standard, but as soon as $C$ and $T_{0}$ are obtained by using a well calibrated magnetometer, the performance of any dc and ac magnetometer may be calibrated by it at any fields and $T$ $>45 \mathrm{~K}$. The standard $\chi$ for the latter two is simple with $\phi=0$, whereas both $|\chi|$ and $\phi$ are functions of frequency and temperature in a complex way when copper cylinder is used. The pure $\mathrm{Dy}_{2} \mathrm{O}_{3}$ powder sample can serve as a primary standard with accuracy on the order of $1 \%$ used at fields below 10 kOe and temperature above $50 \mathrm{~K}$ (see Fig. 5). However, as a standard, a glued powder sample may still be a better choice, since it is easy to handle and to be maintained in a good condition.

Although an overall agreement has been observed between different calibrations, there are remarkable differences found, which leaves the topic of calibration still open.

\section{CONCLUSION}

The performance of ac and dc magnetometers may be calibrated with a glued $\mathrm{Dy}_{2} \mathrm{O}_{3}$ powder sample using its temperature and field dependence of magnetic moment expressed empirically by a combination of the Curie-Weiss law and the Langevin function at $T>45 \mathrm{~K}$. The expressions for the studied sample are Eqs. (2) and (9)-(11), where the Weiss constant $T_{0}=-16.8 \mathrm{~K}$, the normalized elementary magnetic moment $m_{0} / k_{B}=9.8 \times 10^{-4} \mathrm{~K} / \mathrm{Oe}$, and the inverse moment Curie constant $C^{-1}=138.6 \mathrm{Oe} \mathrm{emu}^{-1} \mathrm{~K}^{-1}$. When calibrating ac susceptometers, the measured $|\chi|$ should be compared with Eq. (2) or Eqs. (10) and (11) and the measured $\phi$ should be compared with zero; when calibrating dc magnetometers, the measured $m(H)$ should be compared with Eqs. (9) and (11). Nonzero $\phi$ may occur at lower $T$, indicating a possible phase transition. Without knowing the mass of $\mathrm{Dy}_{2} \mathrm{O}_{3}$, the properties of such glued samples vary between samples. However, pure $\mathrm{Dy}_{2} \mathrm{O}_{3}$ powder samples of known mass may serve as a primary standard for low-field susceptibility, calculated by Eq. (18) with $T_{0}=-17 \mathrm{~K}$, which is a combination of the Curie-Weiss law and the quantum mechanical theory of paramagnetism. The optimum aspect ratio of cylindrical standard samples is 0.85 .

\section{ACKNOWLEDGMENTS}

The authors are grateful to the reviewer for valuable comments and suggestions and to Monica Martinez and Natalia Tristan at Quantum Design for their interest to this study and for providing some samples. Financial support from Consolider Project CSD2007-00041 is acknowledged.

\footnotetext{
${ }^{1}$ Quantum Design, PPMS Hardware \& Options, ACMS User's Manual.

${ }^{2}$ Quantum Design, MPMS Hardware \& Software Reference Manuals.

${ }^{3}$ Quantum Design, Application note 1070-207, Using PPMS Superconducting magnets at low fields (2009).

${ }^{4}$ NBS Standard Reference Materials, Magnetic Gram Susceptibility, 763-Aluminum, 764-Platinum, 765-Palladium, 766-Manganese Fluoride (1973).

${ }^{5}$ D.-X. Chen and V. Skumryev, Rev. Sci. Instrum. 81, 025104 (2010).

${ }^{6}$ S. Chikazumi, Physics of Ferromagnetism (Oxford University Press, Oxford, 1997).

${ }^{7}$ C. P. Bean, Phys. Rev. Lett. 8, 250 (1962).

${ }^{8}$ Y. B. Kim, C. F. Hempstead, and A. R. Strnad, Phys. Rev. Lett. 9, 306 (1962).

${ }^{9}$ D.-X. Chen and R. B. Goldfarb, J. Appl. Phys. 66, 2489 (1989).

${ }^{10} \mathrm{H}$. Bonrath, K. H. Hellwege, K. Nicolay, and G. Weber, Phys. Kondens. Mater. 4, 382 (1966).

${ }^{11}$ A. P. Ramirez, in Handbook of Magnetic Materials, edited by K. H. J. Buschow (North-Holland, Amsterdam, 2001), Vol. 13.

${ }^{12}$ N. Shirakawa, J. E. McArthur, and J. R. O’Brien, J. Phys.: Conf. Ser. 150, 012045 (2009).

${ }^{13}$ V. Skumryev, M. Kuz'min, M. Gospodinov, and J. Fontcuberta, Phys. Rev. B 79, 212414 (2009).

${ }^{14}$ D. J. Flood, Phys. Lett. 49A, 59 (1974).

${ }^{15}$ G. A. Bain and J. F. Berry, J. Chem. Edu. 85, 532 (2008).

${ }^{16}$ M. M. Schieber, Experimental Magnetochemistry, Nonmetallic Magnetic Materials (North-Holland, Amsterdam, 1967) p. 305.

${ }^{17}$ D.-X. Chen, J. A. Brug, and R. B. Goldfarb, IEEE Trans. Magn. 27, 3601 (1991).

${ }^{18}$ D.-X. Chen, Meas. Sci. Technol. 15, 1195 (2004).

${ }^{19}$ P. Stamenov and J. M. D. Coey, Rev. Sci. Instrum. 77, 015106 (2006).
} 